\title{
Localized Waves for the Coupled Mixed Derivative Nonlinear Schrödinger Equation in a Birefringent Optical Fiber
}

\author{
N. Song ${ }^{1}$ - Y. X. Lei ${ }^{1} \cdot$ Y. F. Zhang ${ }^{1} \cdot$ W. Zhang ${ }^{2}$
}

Received: 27 October 2021 / Accepted: 20 February 2022 / Published online: 8 March 2022

(c) The Author(s) 2022

\begin{abstract}
In this paper, the higher-order localized waves for the coupled mixed derivative nonlinear Schrödinger equation are investigated using generalized Darboux transformation. On the basis of seed solutions and a Lax pair, the first- and second-order localized wave solutions are derived from the $N$ th-order iteration formulas of generalized Darboux transformation. Then, the dynamics of the localized waves are analyzed and displayed via numerical simulation. It is found that the second-order rouge wave split into three first-order rogue waves due to the influence of the separation function. In addition, a series of novel dynamic evolution plots exhibit that rogue waves coexist with dark-bright solitons and breathers.
\end{abstract}

Keywords Coupled mixed derivative nonlinear Schrödinger equation · Generalized Darboux transformation $\cdot$ Soliton $\cdot$ Rogue wave $\cdot$ Breather

\section{Introduction}

As is well known, most nonlinear partial differential equations (PDEs) in mathematics and physics are integrable, including the Hirota equation [1], the nonlinear Schrödinger equation [2, 3], the Gerdjikov-Ivanov equation [4], the Korteweg-de Vries equation [5, 6], the Sasa-Satsuma equation [7], and so on. These nonlinear equations play an important role in various fields of nonlinear science such as water waves, nonlinear optics, and Bose-Einstein condensates. Because natural phenomena are more complicated in the real world and in some experimental environments, the study of coupled systems has drawn more attention of many

N. Song

songni@nuc.edu.cn

1 Department of Mathematics, North University of China, Taiyuan 030051, Shanxi, China

2 Faculty of Materials and Manufacturing, Beijing University of Technology, Beijing 100124, China 
researchers than the single-component system [8, 9]. Further, These nonlinear equations can be used to describe and study localized waves, which consist of rogue waves [10, 11], solitons [12, 13], and breathers [14-17]. To date, some methods have been proposed and applied to investigate localized waves of coupled systems, such as the Darboux transformation (DT) [18], the Riemann-Hilbert approach [19], the Hirota bilinear method [20], the generalized DT [21], and so on. Due to the profound theoretical significance and potential applicability, the study of localized waves of the nonlinear PDEs has always been concerned, and great progress has been made [22-29].

In this paper, the coupled mixed derivative nonlinear Schrödinger (CMDNLS) equation is considered [30],

$$
\begin{aligned}
& i q_{1 t}+q_{1 x x}+\mu\left(\left|q_{1}\right|^{2}+\left|q_{2}\right|^{2}\right) q_{1}+i \gamma\left[\left(\left|q_{1}\right|^{2}+\left|q_{2}\right|^{2}\right) q_{1}\right]_{x}=0 \\
& i q_{2 t}+q_{2 x x}+\mu\left(\left|q_{1}\right|^{2}+\left|q_{2}\right|^{2}\right) q_{2}+i \gamma\left[\left(\left|q_{1}\right|^{2}+\left|q_{2}\right|^{2}\right) q_{2}\right]_{x}=0 .
\end{aligned}
$$

In Eq. (1), $q_{1}$ and $q_{2}$ are the slowly varying envelopes of two kinds of polarization in the electric fields, which are the functions of the evolution time $t$ and normalized distance $x . \mu$ and $\gamma$ are real constant parameters that denote the measure of cubic nonlinear strength and derivative cubic nonlinearity, respectively. Eq. (1) can be used to describe the propagation of short pulses in the femtosecond or picosecond regions of birefringent optical fibers. Some results of Eq. (1) have been achieved in Refs. [30-34]. Yan obtained the first-order localized wave solutions via DT and Darboux-dressing transformation, including breather wave solution, rogue wave and bright-dark rogue wave [30]. In the present paper, generalized DT is applied to obtain the $N$ th-order localized waves solutions of Eq. (1) and study the dynamics of the higher-order localized waves.

The remainder of this paper is organized as follows. In Sect. 2, a generalized DT for Eq. (1) is constructed, and the iterative formulas of the Nth-order solutions are deduced. In Sect. 3, the first- and second-order localized wave solitons are given, and some evolution plots of localized wave solitons are illustrated. Finally, several conclusions are provided in Sect. 4.

\section{Generalized Darboux Transformation}

In this section, a generalized DT is constructed and applied to deduce the $N$ thorder localized wave solutions of Eq. (1).

Equation (1) is an integrable nonlinear system, and the Lax pair is given as follows

$$
\begin{gathered}
\Phi_{x}=U \Phi=\left(\lambda^{2} U_{2}+\lambda U_{1}+U_{0}\right) \Phi, \\
\Phi_{t}=V \Phi=\left(\lambda^{4} V_{4}+\lambda^{3} V_{3}+\lambda^{2} V_{2}+\lambda V_{1}+V_{0}\right) \Phi,
\end{gathered}
$$

where 


$$
\begin{aligned}
& U_{0}=-\frac{\mu}{3 \gamma} Q_{0}, U_{1}=\frac{1}{2} Q_{1}, U_{2}=\frac{1}{6 \gamma} Q_{0}, V_{0}=\frac{\mu^{2}}{3 \gamma^{2}} Q_{0}, \\
& V_{1}=-\frac{\mu}{2 \gamma} Q_{1}+\frac{\gamma}{2} Q_{2}+\frac{i}{2} Q_{1 x}, V_{2}=-\frac{\mu}{3 \gamma^{2}} Q_{0}+\frac{i}{4} Q_{3}, V_{3}=\frac{1}{4 \gamma} Q_{1}, V_{4}=\frac{1}{12 \gamma^{2}} Q_{0}, \\
& Q_{0}=\left(\begin{array}{ccc}
-2 i & 0 & 0 \\
0 & i & 0 \\
0 & 0 & i
\end{array}\right), Q_{1}=\left(\begin{array}{ccc}
0 & q_{1} & q_{2} \\
-q_{1}^{*} & 0 & 0 \\
-q_{2}^{*} & 0 & 0
\end{array}\right) Q_{3}=\left(\begin{array}{ccc}
\left|q_{1}\right|^{2}+\left|q_{2}\right|^{2} & 0 & 0 \\
0 & -\left|q_{1}\right|^{2} & -q_{1}^{*} q_{2} \\
0 & -q_{1} q_{2}^{*} & -\left|q_{2}\right|^{2}
\end{array}\right), \\
& Q_{2}=\left(\begin{array}{cc}
0 \\
q_{1}^{*}\left(\left|q_{1}\right|^{2}+\left|q_{2}\right|^{2}\right) & 0 \\
q_{2}^{*}\left(\left|q_{1}\right|^{2}+\left|q_{2}\right|^{2}\right) & 0
\end{array}\right) .
\end{aligned}
$$

Suppose that $\Phi=(\phi, \varphi, \chi)^{T}$ is a vector solution of Eq. (2), $\lambda$ is a spectral parameter and the asterisk denotes the complex conjugate. Thus, it can be easily proven that $U$ and $V$ satisfy the zero-curvature equation $U_{t}-V_{x}+[U, V]=0$.

Through classical DT constructed in Refs. [31, 35], Eq. (2) can be transformed into

$$
\begin{aligned}
\Phi[1]_{x} & =U \Phi[1], \Phi[1]_{t}=V \Phi[1], \\
U[1] & =\left(T_{x}+T U\right) T^{-1}, V[1]=\left(T_{t}+T V\right) T^{-1},
\end{aligned}
$$

where $U[1]$ and $V[1]$ have the same form as $U$ and $V$, meanwhile, the old potentials $q_{1}$ and $q_{2}$ are replaced by the new ones $q_{1}[1]$ and $q_{2}[1]$.

The Darboux matrix $T$ is constructed as follows:

$$
T=\lambda^{2} M_{0}+\lambda M_{1}-I,
$$

where $I$ is a $3 \times 3$ identity matrix, and

$$
\begin{aligned}
M_{0} & =\frac{1}{\lambda_{1}^{* 2}} I+\frac{\lambda_{1}^{* 2}-\lambda_{1}^{2}}{\lambda_{1}^{* 2} \lambda_{1}}\left(\begin{array}{ccc}
\frac{\phi_{1} \phi_{1}^{*}}{\Gamma} & 0 & 0 \\
0 & \frac{\varphi_{1} \varphi_{1}^{*}}{\Gamma^{*}} & \frac{\varphi_{1} \chi_{1}^{*}}{\Gamma^{*}} \\
0 & \frac{\chi_{1} \varphi_{1}^{*}}{\Gamma^{*}} & \frac{\chi_{1} \chi_{1}^{*}}{\Gamma^{*}}
\end{array}\right), M_{1}=\frac{\lambda_{1}^{* 2}-\lambda_{1}^{2}}{\lambda_{1} \lambda_{1}^{*}}\left(\begin{array}{ccc}
0 & \frac{\phi_{1} \varphi_{1}^{*}}{\Gamma} & \frac{\phi_{1} x_{1}^{*}}{\Gamma} \\
\frac{\varphi_{1} \phi_{1}^{*}}{\Gamma^{*}} & 0 & 0 \\
\frac{\chi_{1}^{*} \phi_{1}^{*}}{\Gamma^{*}} & 0 & 0
\end{array}\right), \\
\Gamma & =\lambda_{1}\left|\phi_{1}\right|^{2}+\lambda_{1}^{*}\left|\varphi_{1}\right|^{2}+\lambda_{1}^{*}\left|\chi_{1}\right|^{2} .
\end{aligned}
$$

Assume that $\Phi_{k}=\left(\phi_{k}, \varphi_{k}, \chi_{k}\right)^{T}(k=1,2, \ldots)$ is an eigenfunction of Eq. (2) with $\lambda=\lambda_{k}$. Furthermore, DT can be iterated. Thus, the classical DT of Eq. (1) is defined as follows,

$$
\begin{aligned}
\Phi_{N}[N-1] & =T[N-1] T[N-2] \cdots T[1] T[0] \Phi_{N}, \\
q_{1}[N] & =q_{1}[0]+\sum_{k=1}^{N} 2\left(\lambda_{k}^{* 2}-\lambda_{k}^{2}\right)\left[\frac{i \mu}{\gamma} \frac{\phi_{k}[k-1] \varphi_{k}^{*}[k-1]}{\Lambda_{k}}-\left(\frac{\phi_{k}[k-1] \varphi_{k}^{*}[k-1]}{\Lambda_{k}}\right){ }_{x}\right], \\
q_{2}[N] & =q_{2}[0]+\sum_{k=1}^{N} 2\left(\lambda_{k}^{* 2}-\lambda_{k}^{2}\right)\left[\frac{i \mu}{\gamma} \frac{\phi_{k}[k-1] \chi_{k}^{*}[k-1]}{\Lambda_{k}}-\left(\frac{\phi_{k}[k-1] \chi_{k}^{*}[k-1]}{\Lambda_{k}}\right)_{x}\right], \\
T[0] & =I, T[k]=\lambda_{k+1}^{2} M_{0}[k-1]+\lambda_{k+1} M_{1}[k-1]-I,
\end{aligned}
$$

with 


$$
\begin{aligned}
& \Phi_{k}[k-1]=\left.T[k-1] T[k-2] \cdots T[1]\right|_{\lambda=\lambda_{k}} \Phi_{k}, \\
& \Lambda_{k}=\left|\lambda_{k}\right|^{2}\left[\lambda_{k}\left|\phi_{k}[k-1]\right|^{2}+\lambda_{k}^{*}\left|\varphi_{k}[k-1]\right|^{2}+\lambda_{k}^{*}\left|\chi_{k}[k-1]\right|^{2}\right], \\
& M_{0}[k-1]=\frac{1}{\lambda_{k}^{* 2}} I+\frac{\lambda_{k}^{* 2}-\lambda_{k}^{2}}{\lambda_{k}^{* 2} \lambda_{k}}\left(\begin{array}{ccc}
\frac{\phi_{k}[k-1] \phi_{k}^{*}[k-1]}{\Gamma_{k}} & 0 & 0 \\
0 & \frac{\varphi_{k}[k-1] \varphi_{k}^{*}[k-1]}{\Gamma_{k}^{*}} & \frac{\varphi_{k}[k-1] \chi_{k}^{*}[k-1]}{\Gamma_{k}^{*}} \\
0 & \frac{\chi_{k}[k-1] \varphi_{k}^{*}[k-1]}{\Gamma_{k}^{*}} & \frac{\chi_{k}[k-1] \chi_{k}^{*}[k-1]}{\Gamma_{k}^{*}}
\end{array}\right) \text {, } \\
& M_{1}[k-1]=\frac{\lambda_{k}^{* 2}-\lambda_{k}^{2}}{\lambda_{k} \lambda_{k}^{*}}\left(\begin{array}{ccc}
0 & \frac{\phi_{k}[k-1] \varphi_{k}^{*}[k-1]}{\Gamma_{k}} & \frac{\phi_{k}[k-1] \chi_{k}^{*}[k-1]}{\Gamma_{k}} \\
\frac{\varphi_{k}[k-1] \phi_{k}^{*}[k-1]}{\Gamma_{k}^{*}} & 0 & 0 \\
\frac{\chi_{k}[k-1] \phi_{k}^{*}[k-1]}{\Gamma_{k}^{*}} & 0 & 0
\end{array}\right) \text {, } \\
& \Gamma_{k}=\lambda_{k}\left|\phi_{k}[k-1]\right|^{2}+\lambda_{k}^{*}\left|\varphi_{k}[k-1]\right|^{2}+\lambda_{k}^{*}\left|\chi_{k}[k-1]\right|^{2} .
\end{aligned}
$$

On the basis of above DT, a generalized DT of Eq. (1) can be derived. Assume that $\Phi_{1}=\Phi_{1}\left(\lambda_{1}+\eta\right)=\left(\phi_{1}, \varphi_{1}, \chi_{1}\right)^{T}$ is a solution of Eq. (2) with $q_{1}=q_{1}[0], q_{2}=q_{2}[0]$ and $\lambda=\lambda_{1}+\eta$, and $\eta$ is a small parameter. According to the Taylor expansion of the function $\Phi_{1}$ at $\eta=0, \Phi_{1}$ can be rewritten as follows

$$
\Phi_{1}=\Phi_{1}^{[0]}+\Phi_{1}^{[1]} \eta+\Phi_{1}^{[2]} \eta^{2}+\cdots+\Phi_{1}^{[N]} \eta^{N}+o\left(\eta^{N}\right)
$$

where $\Phi_{1}^{[j]}=\left(\phi_{1}^{[j]}, \varphi_{1}^{[j]}, \chi_{1}^{[j]}\right)^{T}=\left.\frac{1}{j !} \frac{\partial^{j}}{\partial \lambda^{j}} \Phi_{1}(\lambda)\right|_{\lambda=\lambda_{1}}(j=0,1,2, \ldots)$.

It is easy to determine that $\Phi_{1}[0]=\Phi_{1}^{[0]}$ is a vector solution of Eq. (2) with $q_{1}=q_{1}[0], q_{2}=q_{2}[0]$, and $\lambda=\lambda_{1}$. Then, the generalized DT is presented as follows,

$$
\begin{gathered}
\Phi_{1}[N-1]=\Phi_{1}^{[0]}+\sum_{l=1}^{N-1} T_{1}[l] \Phi_{1}^{[1]}+\sum_{l=1}^{N-1} \sum_{k>l}^{N-1} T_{1}[k] T_{1}[l] \Phi_{1}^{[2]}+\cdots \\
+T_{1}[N-1] T_{1}[N-2] \cdots T_{1}[1] \Phi_{1}^{[N-1]}, \\
q_{1}[N]=q_{1}[0]+\sum_{k=1}^{N} 2\left(\lambda_{1}^{* 2}-\lambda_{1}^{2}\right)\left[\frac{i \mu}{\gamma} \frac{\phi_{1}[k-1] \varphi_{1}^{*}[k-1]}{\Lambda_{k}}-\left(\frac{\phi_{1}[k-1] \varphi_{1}^{*}[k-1]}{\Lambda_{k}}\right)_{x}\right], \\
q_{2}[N]=q_{2}[0]+\sum_{k=1}^{N} 2\left(\lambda_{1}^{* 2}-\lambda_{1}^{2}\right)\left[\frac{i \mu}{\gamma} \frac{\phi_{1}[k-1] \chi_{1}^{*}[k-1]}{\Lambda_{k}}-\left(\frac{\phi_{1}[k-1] \chi_{1}^{*}[k-1]}{\Lambda_{k}}\right)_{x}\right], \\
T_{1}[k]=\lambda_{1}^{2} M_{0}[k-1]+\lambda_{1} M_{1}[k-1]-I,(1 \leq k \leq N),
\end{gathered}
$$

with 


$$
\begin{aligned}
& \Phi_{1}[k-1]=\left(\phi_{1}[k-1], \varphi_{1}[k-1], \chi_{1}[k-1]\right)^{T}, \\
& \Lambda_{k}=\left|\lambda_{1}\right|^{2}\left[\lambda_{1}\left|\phi_{1}[k-1]\right|^{2}+\lambda_{1}^{*}\left|\varphi_{1}[k-1]\right|^{2}+\lambda_{1}^{*}\left|\chi_{1}[k-1]\right|^{2}\right] \text {, } \\
& M_{0}[k-1]=\frac{1}{\lambda_{1}^{* 2}} I+\frac{\lambda_{1}^{* 2}-\lambda_{1}^{2}}{\lambda_{1}^{* 2} \lambda_{1}}\left(\begin{array}{ccc}
\frac{\phi_{1}[k-1] \phi_{1}^{*}[k-1]}{\Gamma_{k}} & 0 & 0 \\
0 & \frac{\varphi_{1}[k-1] \varphi_{1}^{*}[k-1]}{\Gamma_{k}^{*}} & \frac{\varphi_{1}[k-1] \chi_{1}^{*}[k-1]}{\Gamma_{k}^{*}} \\
0 & \frac{\chi_{1}[k-1] \varphi_{1}^{*}[k-1]}{\Gamma_{k}^{*}} & \frac{\chi_{1}[k-1] \chi_{1}^{*}[k-1]}{\Gamma_{k}^{*}}
\end{array}\right) \text {, } \\
& M_{1}[k-1]=\frac{\lambda_{1}^{* 2}-\lambda_{1}^{2}}{\lambda_{1} \lambda_{1}^{*}}\left(\begin{array}{ccc}
0 & \frac{\phi_{1}[k-1] \varphi_{1}^{*}[k-1]}{\Gamma_{k}} & \frac{\phi_{1}[k-1] \chi_{1}^{*}[k-1]}{\Gamma_{k}} \\
\frac{\varphi_{1}[k-1] \phi_{1}^{*}[k-1]}{\Gamma_{k}^{*}} & 0 & 0 \\
\frac{\chi_{1}[k-1] \phi_{1}^{*}[k-1]}{\Gamma_{k}^{*}} & 0 & 0
\end{array}\right) \text {, } \\
& \Gamma_{k}=\lambda_{1}\left|\phi_{1}[k-1]\right|^{2}+\lambda_{1}^{*}\left|\varphi_{1}[k-1]\right|^{2}+\lambda_{1}^{*}\left|\chi_{1}[k-1]\right|^{2} \text {. }
\end{aligned}
$$

\section{Dynamics of Localized Waves}

In this section, according to Eqs. (3)-(6), the first- and the second-order localized wave solutions of Eq. (1) are obtained and the dynamics of localized waves are analyzed via numerical simulation plots.

Setting plane waves $q_{1}[0]=d_{1} e^{i \theta}$ and $q_{2}[0]=d_{2} e^{i \theta}$ as the seed solutions of Eq. (1), where $\theta=\mu\left(d_{1}^{2}+d_{2}^{2}\right) t$, and $d_{1}$ and $d_{2}$ are real constants. The corresponding basic solution with $\lambda=\lambda_{1}\left(1+\eta^{2}\right)$ is calculated as follows,

$$
\Phi_{1}=\left(\begin{array}{c}
\left(C_{1} e^{M_{1}+M_{2}}-C_{2} e^{M_{1}-M_{2}}\right) e^{\frac{i}{2} \theta} \\
\rho_{1}\left(C_{1} e^{M_{1}-M_{2}}-C_{2} e^{M_{1}+M_{2}}\right) e^{-\frac{i}{2} \theta}+\alpha d_{2} e^{M_{3}} \\
\rho_{2}\left(C_{1} e^{M_{1}-M_{2}}-C_{2} e^{M_{1}+M_{2}}\right) e^{-\frac{i}{2} \theta}-\alpha d_{1} e^{M_{3}}
\end{array}\right),
$$

where 


$$
\begin{aligned}
C_{1}= & \frac{\left[\frac{\lambda^{2}-2 \mu}{\gamma}-\sqrt{\left(\frac{\lambda^{2}-2 \mu}{\gamma}\right)^{2}+4 \lambda^{2}\left(d_{1}^{2}+d_{2}^{2}\right)}\right]^{\frac{1}{2}}}{\sqrt{\left(\frac{\lambda^{2}-2 \mu}{\gamma}\right)^{2}+4 \lambda^{2}\left(d_{1}^{2}+d_{2}^{2}\right)}, \rho_{1}=\frac{d_{1}}{\sqrt{d_{1}^{2}+d_{2}^{2}}},} \\
C_{2}= & \frac{\left[\frac{\lambda^{2}-2 \mu}{\gamma}+\sqrt{\left(\frac{\lambda^{2}-2 \mu}{\gamma}\right)^{2}+4 \lambda^{2}\left(d_{1}^{2}+d_{2}^{2}\right)}\right]^{\frac{1}{2}}}{\sqrt{\left(\frac{\lambda^{2}-2 \mu}{\gamma}\right)^{2}+4 \lambda^{2}\left(d_{1}^{2}+d_{2}^{2}\right)}, \rho_{2}=\frac{d_{2}}{\sqrt{d_{1}^{2}+d_{2}^{2}}},} \\
M_{1}= & -\frac{i\left(\lambda^{2}-2 \mu\right)}{24 \gamma^{2}\left[2 \gamma x+\left(\lambda^{2}-2 \mu\right) t\right], M_{3}}=\frac{i\left(\lambda^{2}-2 \mu\right)}{12 \gamma^{2}}\left[2 \gamma x+\left(\lambda^{2}-2 \mu\right) t\right], \\
M_{2}= & \frac{i}{8 \gamma} \sqrt{\left(\frac{\lambda^{2}-2 \mu}{\gamma}\right)^{2}+4 \lambda^{2}\left(d_{1}^{2}+d_{2}^{2}\right)\left[2 \gamma x-\left(2 \gamma^{2}\left(d_{1}^{2}+d_{2}^{2}-\lambda^{2}+2 \mu\right)\right) t+\Omega(\eta)\right],} \\
\Omega(\eta)= & \sum_{k=1}^{N} s_{k} \eta^{2 k}, s_{k}=m_{k}+i n_{k}, \quad(k=1,2, \cdots, N) .
\end{aligned}
$$

Here $\alpha, m_{k}$, and $n_{k}$ are real constants. With $\lambda_{1}=i \gamma \sqrt{d_{1}^{2}+d_{2}^{2}}+\sqrt{2 \mu-\gamma^{2}\left(d_{1}^{2}+d_{2}^{2}\right)}$, expanding function $\Phi_{1}(\eta)$ as Taylor series in the neighborhood of $\eta=0$,

$$
\Phi_{1}=\Phi_{1}^{[0]}+\Phi_{1}^{[1]} \eta^{2}+\Phi_{1}^{[2]} \eta^{4}+\cdots+\Phi_{1}^{[j]} \eta^{2 j}+\cdots,(j=0,1,2, \ldots)
$$

where $\Phi_{1}^{[j]}$ can be obtained with Maple, and

$$
\begin{aligned}
\phi_{1}^{[0]} & =-\frac{-\sqrt{\delta} \sqrt{-\delta \gamma^{2}+2 \mu}(3 \delta \gamma t-x)-3 i \delta^{2} \gamma^{2} t+i \delta \gamma x+2 i \delta \mu t+1}{\left(2 i \sqrt{\delta} \sqrt{-\delta \gamma^{2}+2 \mu}-2 \delta \gamma\right)^{\frac{1}{2}}} e^{\xi_{1}}, \\
\varphi_{1}^{[0]} & =\frac{-\sqrt{\delta} \sqrt{-\delta \gamma^{2}+2 \mu}(3 \delta \gamma t-x)-3 i \delta^{2} \gamma^{2} t+i \delta \gamma x+2 i \delta \mu t-1}{\sqrt{\delta}\left(2 i \sqrt{\delta} \sqrt{-\delta \gamma^{2}+2 \mu}-2 \delta \gamma\right)^{\frac{1}{2}}} d_{1} e^{\xi_{2}}+\alpha d_{2} e^{\xi_{3}}, \\
\chi_{1}^{[0]} & =\frac{-\sqrt{\delta} \sqrt{-\delta \gamma^{2}+2 \mu}(3 \delta \gamma t-x)-3 i \delta^{2} \gamma^{2} t+i \delta \gamma x+2 i \delta \mu t-1}{\sqrt{\delta}\left(2 i \sqrt{\delta} \sqrt{-\delta \gamma^{2}+2 \mu}-2 \delta \gamma\right)^{\frac{1}{2}}} d_{2} e^{\xi_{2}}-\alpha d_{1} e^{\xi_{3}},
\end{aligned}
$$

with $\delta=d_{1}^{2}+d_{2}^{2}$, and 


$$
\begin{aligned}
& \xi_{1}=-\frac{1}{3} \sqrt{\delta} \sqrt{-\delta \gamma^{2}+2 \mu}\left(\delta \gamma t-\frac{1}{2} x\right)+\frac{1}{6} i \delta\left(-2 \delta \gamma^{2} t+\gamma x+5 \mu t\right), \\
& \xi_{2}=-\frac{1}{3} \sqrt{\delta} \sqrt{-\delta \gamma^{2}+2 \mu}\left(\delta \gamma t-\frac{1}{2} x\right)-\frac{1}{6} i \delta\left(2 \delta \gamma^{2} t-\gamma x+\mu t\right), \\
& \xi_{3}=\frac{1}{3} i\left(i \sqrt{\delta} \sqrt{-\delta \gamma^{2}+2 \mu} t-\delta \gamma t+x\right)\left(i \sqrt{\delta} \sqrt{-\delta \gamma^{2}+2 \mu}-\delta \gamma\right) .
\end{aligned}
$$

Moreover, the expressions of $\phi_{1}^{[1]}, \varphi_{1}^{[1]}$, and $\chi_{1}^{[1]}$ are omitted due to their cumbersome forms.

Obviously, $\Phi_{1}[0]=\Phi_{1}^{[0]}=\left(\phi_{1}^{[0]}, \varphi_{1}^{[0]}, \chi_{1}^{[0]}\right)^{T}$ is a solution of Eq. (2) with $q_{1}[0]=d_{1} e^{i \theta}, q_{2}[0]=d_{2} e^{i \theta}$, and $\lambda=\lambda_{1}$. According Eqs. (5)-(7), the first-order localized wave solutions of Eq. (1) are obtained,

$$
\begin{aligned}
& q_{1}[1]=q_{1}[0]+2\left(\lambda_{1}^{* 2}-\lambda_{1}^{2}\right)\left[\frac{i \mu}{\gamma} \frac{\phi_{1}[0] \varphi_{1}^{*}[0]}{\Lambda_{1}}-\left(\frac{\phi_{1}[0] \varphi_{1}^{*}[0]}{\Lambda_{1}}\right)_{x}\right], \\
& q_{2}[1]=q_{2}[0]+2\left(\lambda_{1}^{* 2}-\lambda_{1}^{2}\right)\left[\frac{i \mu}{\gamma} \frac{\phi_{1}[0] \chi_{1}^{*}[0]}{\Lambda_{1}}-\left(\frac{\phi_{1}[0] \chi_{1}^{*}[0]}{\Lambda_{1}}\right)_{x}\right],
\end{aligned}
$$

with

$$
\Lambda_{1}=\left|\lambda_{1}\right|^{2}\left[\lambda_{1}\left|\phi_{1}[0]\right|^{2}+\lambda_{1}^{*}\left|\varphi_{1}[0]\right|^{2}+\lambda_{1}^{*}\left|\chi_{1}[0]\right|^{2}\right]
$$

There are five free parameters $\mu, \gamma, d_{1}, d_{2}$, and $\alpha$, included in solutions $q_{1}[1]$ and $q_{2}[1]$. With the change of free parameters, the dynamic behaviors of the first-order localized wave solutions are analyzed in three cases.

(1) $d_{1}=d_{2}=1$ and $\alpha=0$. The first-order rogue waves $q_{1}[1]$ and $q_{2}[1]$ have the spatial and temporal symmetry, which is similar to those reported in Ref. [30].

(2) $d_{1}=1, d_{2}=0$ and $\alpha \neq 0$. Figures 1 and 2 exhibit the interaction between a darkbright soliton and a rogue wave. With the parameter $\alpha=\frac{1}{10}$, the dark-bright

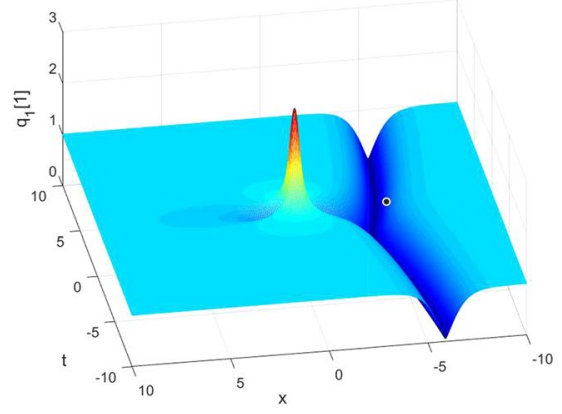

(a)

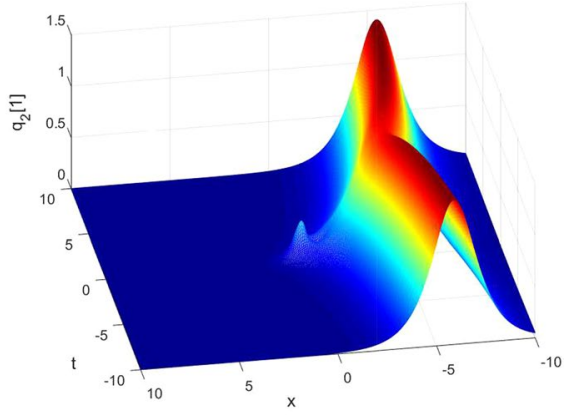

(b)

Fig. 1 The first-order localized waves with parameters $\mu=2, \gamma=\frac{1}{100}, d_{1}=1, d_{2}=0$, and $\alpha=\frac{1}{10}$ 


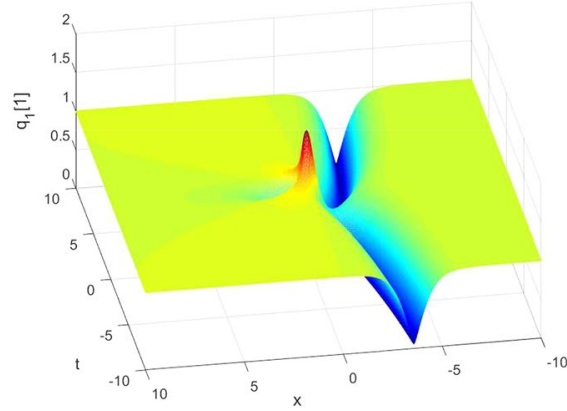

(a)

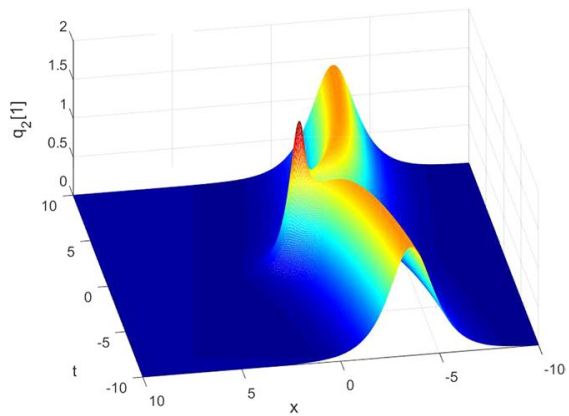

(b)

Fig. 2 The first-order localized waves with parameters $\mu=2, \gamma=\frac{1}{100}, d_{1}=1, d_{2}=0$, and $\alpha=1$

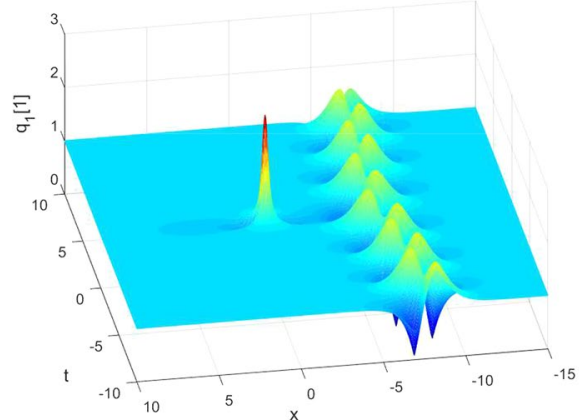

(a)

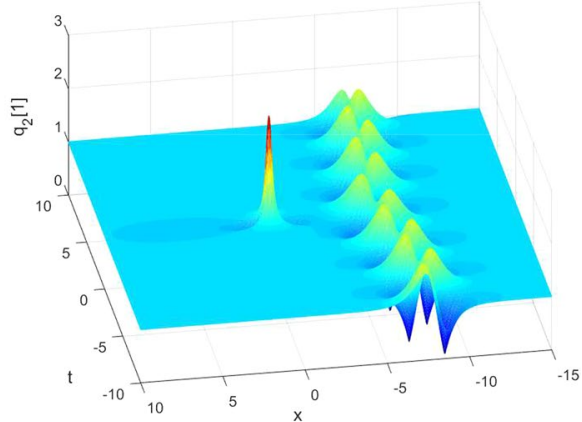

(b)

Fig. 3 The first-order localized waves with parameters $\mu=2, \gamma=\frac{1}{100}, d_{1}=d_{2}=1$, and $\alpha=\frac{1}{1000}$

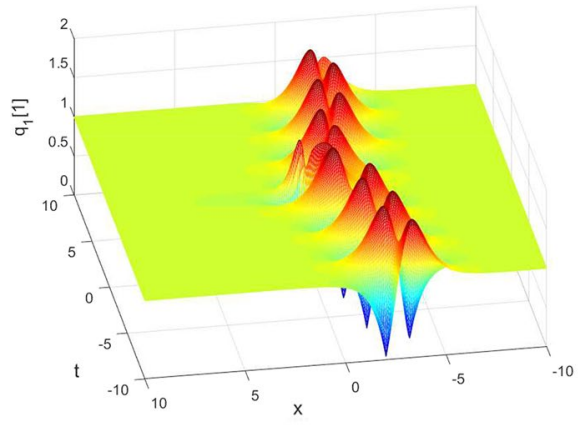

(a)

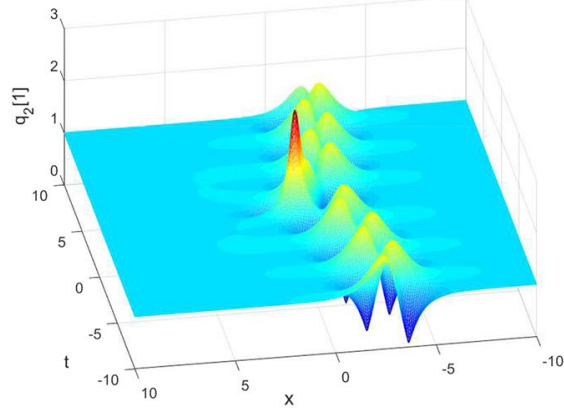

(b)

Fig. 4 The first-order localized waves with parameters $\mu=2, \gamma=\frac{1}{100}, d_{1}=d_{2}=1$, and $\alpha=1$ 
soliton together with the rogue wave in the component $q_{1}[1]$ can be observed, but in the component $q_{2}$ [2], the rogue wave cannot be easily identified, as demonstrated in Fig. 1. Moreover, it is found from Figs. 1 and 2 that the dark-bright soliton and the rogue wave merge with increases in the value of $\alpha$.

(3) $d_{1}=d_{2}=1$ and $\alpha \neq 0$. The interaction between a breather and a rogue wave is demonstrated in Figs. 3 and 4. It is found that the propagation direction of a breather is parallel with the positive direction of t-axis. Compared Figs. 4 with 3 , from which it is also observed that the breather and the rogue wave merge with increases in the value of $\alpha$.

Next, considering the following limit,

$$
\Phi_{1}[1]=\lim _{\eta \rightarrow 0} \frac{\left.T[1]\right|_{\lambda=\lambda_{1}\left(1+\eta^{2}\right)} \Phi_{1}}{\eta^{2}}=\lambda_{1} \Phi_{1}^{[0]}+T_{1}[1] \Phi_{1}^{[1]},
$$

where

$$
\begin{aligned}
& \Phi_{1}[1]=\left(\phi_{1}[1], \varphi_{1}[1], \chi_{1}[1]\right)^{T}, \\
& T_{1}[1]=\lambda_{1}^{2} M_{0}[0]+\lambda_{1} M_{1}[0]-I \text {, } \\
& M_{0}[0]=\frac{1}{\lambda_{1}^{* 2}} I+\frac{\lambda_{1}^{* 2}-\lambda_{1}^{2}}{\lambda_{1}^{* 2} \lambda_{1}}\left(\begin{array}{ccc}
\frac{\phi_{1}[0] \phi_{1}^{*}[0]}{\Gamma_{1}} & 0 & 0 \\
0 & \frac{\varphi_{1}[0] \varphi_{1}^{*}[0]}{\Gamma_{1}^{*}} & \frac{\varphi_{1}[0] \chi_{1}^{*}[0]}{\Gamma_{1}^{*}} \\
0 & \frac{\chi_{1}[0] \varphi_{1}^{*}[0]}{\Gamma_{1}^{*}} & \frac{\chi_{1}[0] \chi_{1}^{*}[0]}{\Gamma_{1}^{*}}
\end{array}\right) \text {, } \\
& M_{1}[0]=\frac{\lambda_{1}^{* 2}-\lambda_{1}^{2}}{\lambda_{1} \lambda_{1}^{*}}\left(\begin{array}{ccc}
0 & \frac{\phi_{1}[0] \varphi_{1}^{*}[0]}{\Gamma_{1}} & \frac{\phi_{1}[0] \chi_{1}^{*}[0]}{\Gamma_{1}} \\
\frac{\varphi_{1}[0] \phi_{1}^{*}[0]}{\Gamma_{1}^{*}} & 0 & 0 \\
\frac{\chi_{1}[0] \phi_{1}^{*}[0]}{\Gamma_{1}^{*}} & 0 & 0
\end{array}\right) \text {, } \\
& \Gamma_{1}=\lambda_{1}\left|\phi_{1}[0]\right|^{2}+\lambda_{1}^{*}\left|\varphi_{1}[0]\right|^{2}+\lambda_{1}^{*}\left|\chi_{1}[0]\right|^{2} \text {. }
\end{aligned}
$$

According Eqs. (5)-(8), the second-order localized wave solutions of Eq. (1) are obtained with the free parameters $\mu, \gamma, d_{1}, d_{2}, \alpha, m_{1}$, and $n_{1}$,

$$
\begin{aligned}
& q_{1}[2]=q_{1}[1]+2\left(\lambda_{1}^{* 2}-\lambda_{1}^{2}\right)\left[\frac{i \mu}{\gamma} \frac{\phi_{1}[1] \varphi_{1}^{*}[1]}{\Lambda_{2}}-\left(\frac{\phi_{1}[1] \varphi_{1}^{*}[1]}{\Lambda_{2}}\right)_{x}\right], \\
& q_{2}[2]=q_{2}[1]+2\left(\lambda_{1}^{* 2}-\lambda_{1}^{2}\right)\left[\frac{i \mu}{\gamma} \frac{\phi_{1}[1] \chi_{1}^{*}[1]}{\Lambda_{2}}-\left(\frac{\phi_{1}[1] \chi_{1}^{*}[1]}{\Lambda_{2}}\right)_{x}\right],
\end{aligned}
$$

with

$$
\Lambda_{2}=\left|\lambda_{1}\right|^{2}\left[\lambda_{1}\left|\phi_{1}[1]\right|^{2}+\lambda_{1}^{*}\left|\varphi_{1}[1]\right|^{2}+\lambda_{1}^{*}\left|\chi_{1}[1]\right|^{2}\right]
$$

Similarly, the dynamic properties of the second-order localized wave solutions with the variations of the free parameters involved are discussed in following cases. 


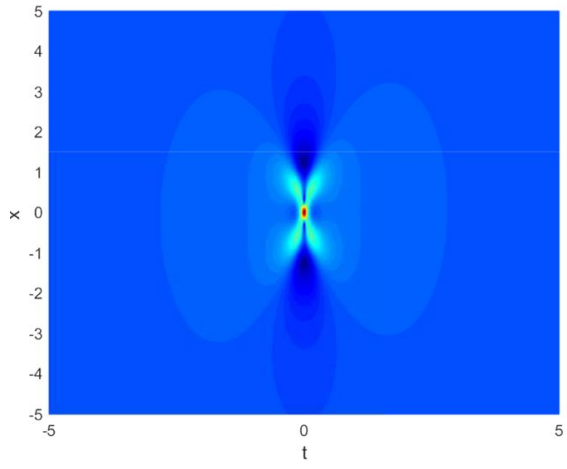

(a) $m_{1}=0$

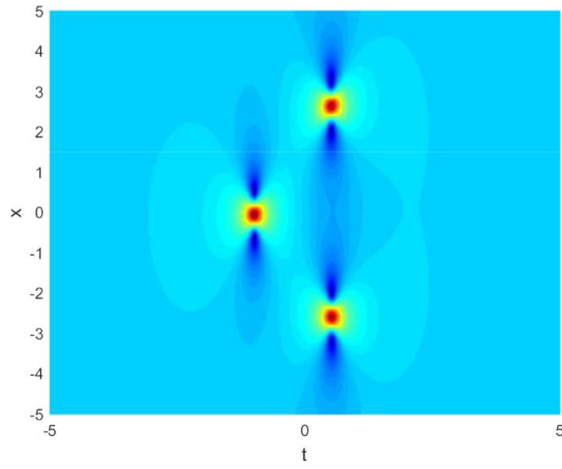

(b) $m_{1}=100$

Fig. 5 The second-order rogue waves with parameters $\mu=2, \gamma=\frac{1}{100}, d_{1}=d_{2}=1, \alpha=0$, and $n_{1}=0$

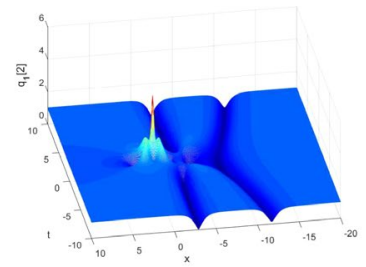

(a) $\alpha=\frac{1}{10}, m_{1}=0$

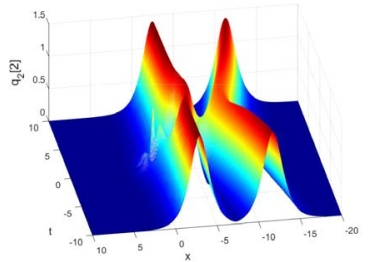

(d) $\alpha=\frac{1}{10}, m_{1}=0$

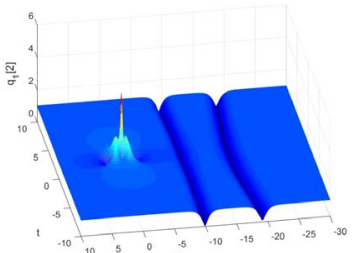

(b) $\alpha=\frac{1}{10000}, m_{1}=0$

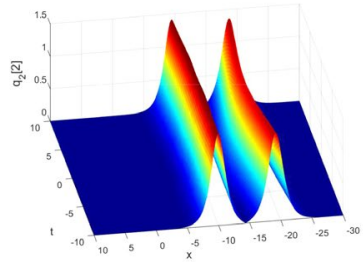

(e) $\alpha=\frac{1}{10000}, m_{1}=0$

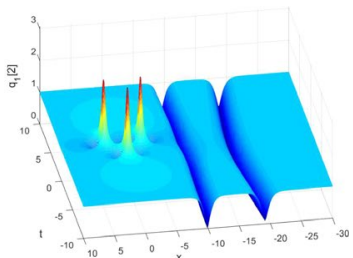

(c) $\alpha=\frac{1}{10000}, m_{1}=100$

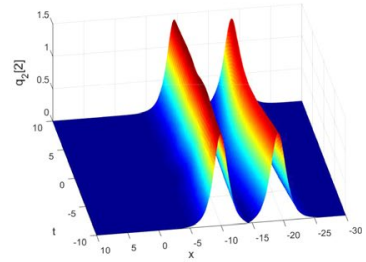

(f) $\alpha=\frac{1}{10000}, m_{1}=100$

Fig. 6 The second-order localized waves with parameters $\mu=2, \gamma=\frac{1}{100}, d_{1}=1, d_{2}=0$, and $n_{1}=0$

(1) $d_{1}=d_{2}=1$ and $\alpha=0$. Figure 5 presents the contour plots of components $q_{1}$ [2] and $q_{2}[2]$. Let $m_{1}=0, q_{1}[2]$ and $q_{2}$ [2] are the fundamental second-order rogue wave in Fig. 5a. Furthermore, setting $m_{1}=100, q_{1}[2]$ and $q_{2}$ [2] are consist of three first-order rogue waves in Fig. $5 b$.

(2) $d_{1}=1, d_{2}=0$ and $\alpha \neq 0$. The interactions of two dark-bright solitons and a second-order rogue wave are illustrated in Fig. 6. Similarly to dynamics of the second-order localized waves in Figs. 1 and 2, the second-order rogue wave in the component $q_{1}$ [2] can be clearly observed, but in the component $q_{2}$ [2], the rogue wave cannot be easily identified after setting $\alpha=\frac{1}{10000}$, as demonstrated in Fig. 6. Decreasing the value of $\alpha$, the second-order rogue wave separates from two dark-bright solitons. In addition, With the parameter $m_{1}=100$, the funda- 


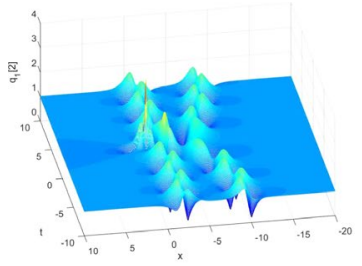

(a) $\alpha=\frac{1}{10}, m_{1}=0$

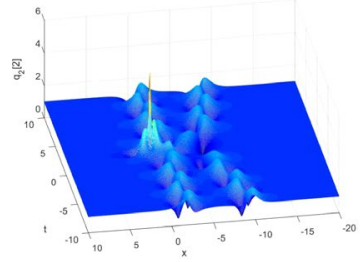

(d) $\alpha=\frac{1}{10}, m_{1}=0$

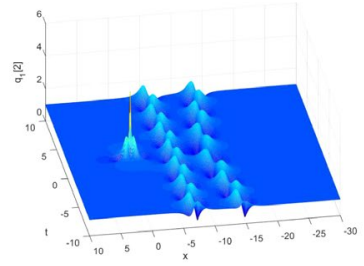

(b) $\alpha=\frac{1}{10000}, m_{1}=0$

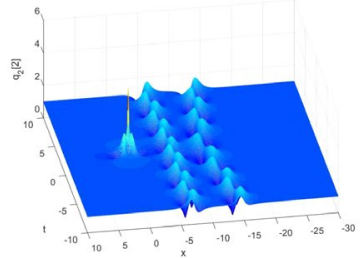

(e) $\alpha=\frac{1}{10000}, m_{1}=0$

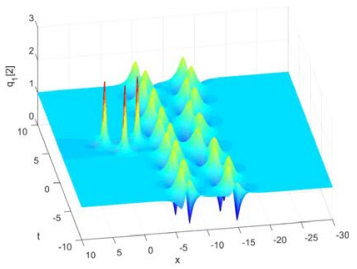

(c) $\alpha=\frac{1}{10000}, m_{1}=100$

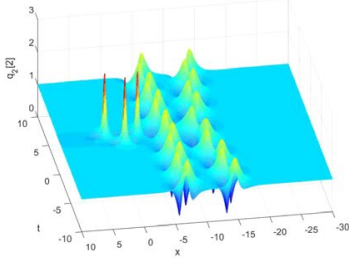

(f) $\alpha=\frac{1}{10000}, m_{1}=100$

Fig. 7 The second-order localized waves with parameters $\mu=2, \gamma=\frac{1}{100}, d_{1}=d_{2}=1$, and $n_{1}=0$

mental second-order rogue wave can split into three first-order rogue waves in Fig. $6 \mathrm{c}$ and $\mathrm{f}$.

(3) $d_{1}=d_{2}=1$ and $\alpha \neq 0$. Figure 7 demonstrates a second-order rogue wave coexisted with breathers. It is found that the propagation directions of a breathers are parallel with the positive direction of t-axis, and also observed that breathers and the second-order rogue wave separate with decreases in the value of $\alpha$. Moreover, due to the influence of separation function $\Omega(\eta)$, the fundamental second-order rogue wave splits into three first-order rogue waves, as displayed in Fig. 7c and f.

\section{Conclusions}

The CMDNLS equation was studied in this work. Based on the classical DT and a Lax pair, a generalized DT was constructed and the $N$ th-order localized wave solutions were derived. It is found that the free parameters involved in localized wave solutions are $\mu, \gamma, \alpha, d_{1}, d_{2}, m_{j}$ and $n_{j}(j=1,2, \ldots)$. Then, the first- and second-order localized wave solutions were obtained and the corresponding evolution plots were provided. Further, altering the values of related parameters, the 
interactions of localized waves were analyzed. The results of this paper present richer dynamics of CMDNLS equation.

Funding The authors gratefully acknowledge the support of the National Natural Science Foundation of China (NNSFC) through grant Nos. 11602232, the Graduate Innovation Project Fund of Shanxi Province through grant Nos. 2021Y629, and the Fund for Shanxi 1331KIRT.

Availability of data and material No data, models, or code were generated or used during the study.

\section{Declarations}

Conflict of interests The authors declare that they have no conflict of interest.

Open Access This article is licensed under a Creative Commons Attribution 4.0 International License, which permits use, sharing, adaptation, distribution and reproduction in any medium or format, as long as you give appropriate credit to the original author(s) and the source, provide a link to the Creative Commons licence, and indicate if changes were made. The images or other third party material in this article are included in the article's Creative Commons licence, unless indicated otherwise in a credit line to the material. If material is not included in the article's Creative Commons licence and your intended use is not permitted by statutory regulation or exceeds the permitted use, you will need to obtain permission directly from the copyright holder. To view a copy of this licence, visit http://creativecommons.org/licen ses/by/4.0/.

\section{References}

1. Xie, X.Y., Liu, X.B.: Elastic and inelastic collisions of the semirational solutions for the coupled Hirota equations in a birefringent fiber. Appl. Math. Lett. 105, 106291 (2020)

2. Bilman, D., Buckingham, R.: Large-order asymptotics for multiple-pole solitons of the focusing nonlinear Schrödinger equation. J. Nonlinear Sci. 29, 2185-2229 (2019)

3. Bilman, D., Buckingham, R., Wang, D.S.: Far-field asymptotics for multiple-pole solitons in the large-order limit. J. Differ. Equ. 297, 320-369 (2021)

4. Wu, J.P.: Integrability aspects and multi-soliton solutions of a new coupled Gerdjikov-Ivanov derivative nonlinear Schrödinger equation. Nonlinear Dyn. 96, 789-800 (2019)

5. Ankiewicz, A., Bokaeeyan, M., Akhmediev, N.: Shallow-water rogue waves: An approach based on complex solutions of the Korteweg-de Vries equation. Phys. Rev. E 99, 050201 (2019)

6. Wang, D.S., Xu, L., Xuan, Z.X.: The complete classification of solutions to the Riemann problem of the defocusing complex modified KdV equation. J. Nonlinear Sci. 32, 3 (2022)

7. Tantawy, M., Abdel-Gawad, H.I.: On multi-geometric structures optical waves propagation in self-phase modulation medium: Sasa-Satsuma equation. Eur. Phys. J. Plus 135, 928-937 (2020)

8. Baronio, F., Degasperis, A., Conforti, M., Wabnitz, S.: Solutions of the vector nonlinear Schrödinger equations: evidence for deterministic rogue waves. Phys. Rev. Lett. 109, 044102 (2012)

9. Baizakov, B.B., Bouketir, A., Al-Marzoug, S.M., Bahlouli, H.: Effect of quintic nonlinearity on modulation instability in coupled nonlinear Schrödinger systems. Optik 180, 792-798 (2019)

10. Draper, L.: Freak ocean waves. Mar. Obs. 35, 193-195 (1965)

11. Li, Z.D., Huo, C.Z., Li, Q.Y., He, P.B., Xu, T.F.: Symmetry and asymmetry rogue waves in twocomponent coupled nonlinear Schrödinger equations. Chin. Phys. B 27, 040505 (2018)

12. Zabusky, N.J., Kruskal, M.D.: Interaction of "solitons"' in a collisionless plasma and the recurrence of initial states. Phys. Rev. Lett. 15, 240-243 (1965)

13. Qin, Y.H., Zhao, L.C., Yang, Z.Q., Ling, L.M.: Multivalley dark solitons in multicomponent BoseEinstein condensates with repulsive interactions. Phys. Rev. E 104, 014201 (2021)

14. Akhmediev, N.N., Korneev, V.I.: Modulation instability and periodic solutions of the nonlinear Schrödinger equation. Theor. Math. Phys. 69, 1089-1093 (1986) 
15. Kuznetsov, E.A.: Solitons in parametrically unstable plasma. Doklady Akademii Nauk SSSR 236, 575-577 (1977)

16. Ma, Y.C.: The Perturbed plane-wave solutions of the cubic Schrödinger equation. Stud. Appl. Math. 60, 43-58 (1979)

17. Sullivan, J., Charalampidis, E.G., Cuevas-Maraver, J., Kevrekidis, P.G., Karachalios, N.I.: Kuznetsov-Ma breather-like solutions in the Salerno model. Eur. Phys. J. Plus 135, 607-618 (2020)

18. Shi, X.J., Lv, P., Qi, C.: Explicit solutions to a nonlocal 2-component complex modified Kortewegde Vries equation. Appl. Math. Lett. 100, 106043 (2020)

19. Kang, Z.Z., Xia, T.C., Ma, X.: Multi-soliton solutions for the coupled modified nonlinear Schrödinger equations via Riemann-Hilbert approach. Chin. Phys. B 27, 070201 (2018)

20. Liu, X.Y., Triki, H., Zhou, Q., Mirzazadeh, M., Liu, W.J., Biswas, A., Belic, M.: Generation and control of multiple solitons under the influence of parameters. Nonlinear Dyn. 95, 143-150 (2019)

21. Song, N., Zhao, X.Y., Shi, N.: Generalized Darboux transformation and nonlinear analysis of higher-order localized wave solutions. Int. J. Dyn. Control 9, 1396-1401 (2021)

22. Zhang, Y.S., Cheng, Y., He, J.S.: Riemann-Hilbert method and N-soliton for two-component Gerdjikov-Ivanov equation. J. Nonlinear Math. Phys. 24, 210-223 (2017)

23. Wazwaz, A.M.: Optical bright and dark soliton solutions for coupled nonlinear Schrödinger (CNLS) equations by the variational iteration method. Optik 207, 164457 (2020)

24. Feng, B.F., Ling, L.M., Takahashi, D.A.: Multi-breather and high-order rogue waves for the nonlinear Schrödinger equation on the elliptic function background. Stud. Appl. Math. 144, 46-101 (2020)

25. Song, N., Xue, H., Xue, Y.K.: Dynamics of higher-order localized waves for a coupled nonlinear Schrödinger equation. Commun. Nonlinear Sci. Numer. Simul. 82, 105046 (2020)

26. Sudhir Singh, K., Sakkaravarthi, K. Murugesan., Sakthivel, R.: Benjamin-Ono equation: Rogue waves, generalized breathers, soliton bending, fission, and fusion. Eur. Phys. J. Plus 135, 823-840 (2020)

27. Wang, D.S., Shi, Y.R., Feng, W.X., Wen, L.: Dynamical and energetic instabilities of F=2 spinor Bose-Einstein condensates in an optical lattice. Phys. D Nonlinear Phenom. 351, 30-41 (2017)

28. Wang, D.S., Zhang, D.J., Yang, J.K.: Integrable properties of the general coupled nonlinear Schrödinger equations. J. Math. Phys. 51, 23510 (2010)

29. Wang, D.S., Guo, B.L., Wang, X.L.: Long-time asymptotics of the focusing Kundu-Eckhaus equation with nonzero boundary conditions. J. Differ. Equ. 266, 5209-5253 (2019)

30. Yan, X.W.: Lax pair, Darboux-dressing transformation and localized waves of the coupled mixed derivative nonlinear Schrödinger equation in a birefringent optical fiber. Appl. Math. Lett. 107, $106414(2020)$

31. Zhang, H.Q.: Darboux transformation and n-soliton solution for the coupled modified nonlinear Schrödinger equations. Zeitschrift für Naturforschung A 67, 711-722 (2012)

32. Hang, C., Wu, Q.L., Zhang, H.Q.: Breathers and double-pole solutions of coupled mixed derivative nonlinear Schrödinger equations from optical fibers. Mod. Phys. Lett. B 35, 2150373 (2021)

33. Hisakado, M., Iizuka, T., Wadati, M.: Coupled hybrid nonlinear Schrödinger equation and optical solitons. J. Phys. Soc. Jpn. 63, 2887-2894 (1994)

34. Li, M., Xiao, J.H., Qin, B., Wang, M., Tian, B.: Vector-soliton bound states for the coupled mixed derivative nonlinear Schrödinger equations in optical fibers. Wave Motion 50, 1-10 (2013)

35. Gu, C.H., Hu, H.S., Zhou, Z.X.: Darboux Transformations in Integrable Systems: Theory and their Applications to Geometry. Springer, New York (2005) 